\title{
Origins of HTLV-1 in South America
}

To the editor-In the December 1999 issue, Li et al. provided a genetic description of a previously unknown strain of HTLV-1 isolated from an Andean mummy dated approximately 1,500 years old ${ }^{1}$. Examining polymorphic sites within small fragments of the LTR (157 base pairs) and $\mathrm{pX}$ regions (159 base pairs) a simple comparison of the mummy with modern-day Chilean and Japanese isolates of HTLV-1, led to the conclusion that the mummy strain was carried into the New World during the ancestral migrations of humans from Asia across the Bering Strait. Although intriguing, the authors' conclusion is in contrast to the preponderance of data that indicate a far more recent origin for HTLV-1 within South America $^{2-6}$.

For several years, we and others examined the origin and evolutionary history of HTLV-1 through molecular phylogenetic analyses and historical and paleoanthropological data ${ }^{2-5}$. These analyses consistently define distinct evolutionary lineages within type 1 viruses, including the Cosmopolitan clade studied in the paper by Li et $a l^{1}$. The prevailing hypothesis is that the assemblage of American HTLV-1 strains within the Cosmopolitan clade originated in Africa, and were disseminated by the slave trade that occurred during the colonial era.

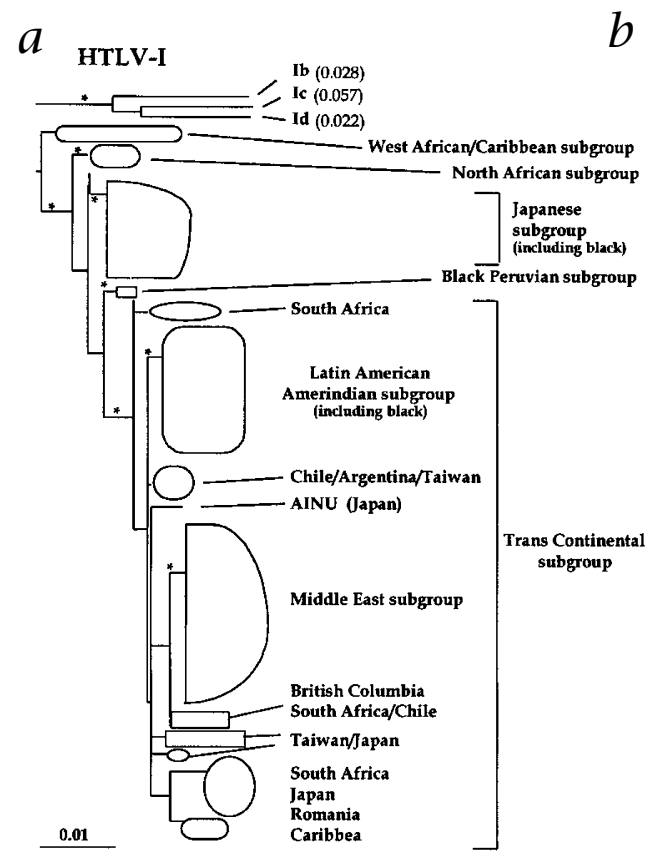

A careful consideration of the following points, combined with the results of our phylogenetic analysis of these previously unknown Andean mummy sequences, does not support the authors' claim that these sequences have an ancestral Asian origin. All major endemic groups infected with HTLV-1 in the New World consist of ethnic populations of African descent ${ }^{6}$. In addition, HTLV-1 infection is nearly absent in most native American Indians. For those rare American-Indian HTLV-1 strains available, phylogenetic analyses show close association with strains isolated from individuals of African descent living in the Americas and the Caribbean as well as those from the western region of Africa $^{3,5,6}$. Moreover, within the Cosmopolitan clade, HTLV-1 subdivides into four main groupings. Each of these is at least affiliated with an HTLV-1 that was isolated from individuals of African descent, which indicates an African ori$\operatorname{gin}^{3-6}$. Likewise, under the hypothesis of a 'molecular clock', evidence for a more recent post-Columbian introduction of HTLV-1 into the Americas is strongly suggested $^{3}$. Finally, we did a phylogenetic assessment of the mummy long terminal repeat segment ( 157 base pairs) relative to all known diverse representatives of HTLV-1 (ref. 5). Although the mummy sequences cluster within the Cosmopolitan clade, they cannot be re-

solved further into subgroups because of the lack of informative mutations in the small region analyzed. Our results, therefore, show no evidence of an Asian origin for the mummy strain. Moreover, the mummy sequences do not have a basal position within the Cosmopolitan clade, as would be expected if they were ancestral to the other component strains.

Although we recognize that there are many unanswered questions concerning the patterns of dissemination of the Cosmopolitan HTLV-1 strains, the most parsimonious interpretation of all the data is that the Andean mummy strain is modern in origin.

Antoine Gessain ${ }^{1}$, Jill Pecon-

Slattery $^{2}$, Laurent MeERTens ${ }^{1} \&$

RENAUD MAHIEUX ${ }^{1}$

'Unité d'Oncologie Virale

Département du SIDA et des Rétrovirus. Institut Pasteur, 25-28 rue du Dr. Roux 75724 Paris Cedex 15,

France

${ }^{2}$ Laboratory of Genomic Diversity Frederick Cancer and Research Development Center, National Cancer Institute Frederick, Maryland 21702, USA

Correspondence should be sent to A.G.; Email: agessain@pasteur.fr

To the editor-Li et al. describe the presence of HTLV-I proviral DNA in one of two globin-positive archival samples isolated from 1,500-year-old Andean mummies ${ }^{1}$, indicating a prevalence of infection of around 50\% at that time. However, the current prevalence of HTLV-I in American

$\mathrm{d}\left(\mathrm{Ib}, \mathrm{sp} 2^{\mathrm{a}}\right)$

$\mathrm{d}\left(\mathrm{Ib}\right.$, ATK $\left.^{\mathrm{b}}\right)$

$\mathrm{d}\left(\mathrm{Ib}, \mathrm{H} 5^{\mathrm{b}}\right)$

$\mathrm{d}\left(\mathrm{Ib}, \mathrm{KAG} 130^{\mathrm{b}}\right)$

$\mathrm{d}\left(\mathrm{Ib}, \mathrm{CHI} 430^{\mathrm{c}}\right)$

$\mathrm{d}\left(\mathrm{Ib}, \mathrm{CHI} 383^{\mathrm{c}}\right)$

aHTLV-la from mummy? 'bStrains belonging to the Japanese clade. 'Chilean strains.

Fig. 1 Genetic variability in Cosmopolitan HTLV-1. a, Neighbor-joining tree of 109 HTLVla strains, with HTLV-Ib, Ic, and Id as outgroups, using a 512-nucleotide fragment of the long terminal repeat region. Branch lengths (in parentheses for the outgroups) are optimized using the maximum likelihood method. ${ }^{*}, P<$ 0.01. b, Genetic distance between HTLV-lb (outgroup) and different HTLV-la strains calculated with the HKY85 model. Underlining, distances from present-day strains to the outgroup smaller than the distance of the 'putative' mummy sample from the outgroup.
Indians from Latin America ranges from $0 \%$ to $15 \%$ (ref. 7 ).

All HTLV-I subtypes have originated through separate simian-tohuman transmissions in Asia and Africa $^{8}$. The Cosmopolitan subtype originated in Africa and spread worldwide, including to Japan and Latin-America. The largest genetic variability of Cosmopolitan HTLV-1 is found in Black populations which carry strains found in all five subgroups (Fig. 1a). Although most Japanese strains cluster very tightly in just one subgroup (the Japanese subgroup), some also belong to the Transcontinental subgroup. In the Transcontinental subgroup, virus strains from Black populations have a larger genetic vari- 